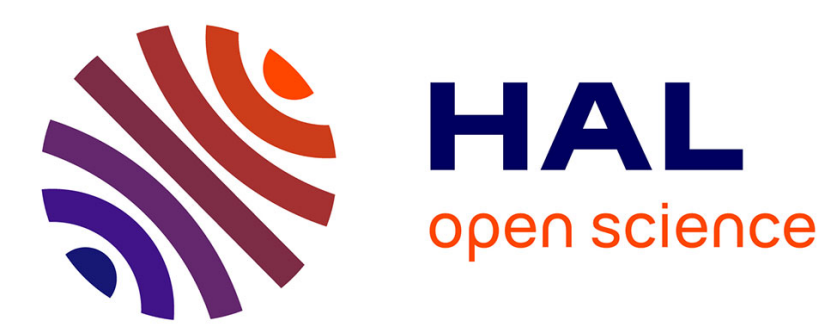

\title{
Fault detection and identification relying on set-membership identifiability
}

Carine Jauberthie, Nathalie Verdière, Louise Travé-Massuyès

\section{To cite this version:}

Carine Jauberthie, Nathalie Verdière, Louise Travé-Massuyès. Fault detection and identification relying on set-membership identifiability. Annual Reviews in Control, 2013, 37 (1), pp.129 - 136. 10.1016/j.arcontrol.2013.04.002 . hal-01739792

\section{HAL Id: hal-01739792 \\ https://hal.science/hal-01739792}

Submitted on 21 Nov 2018

HAL is a multi-disciplinary open access archive for the deposit and dissemination of scientific research documents, whether they are published or not. The documents may come from teaching and research institutions in France or abroad, or from public or private research centers.
L'archive ouverte pluridisciplinaire HAL, est destinée au dépôt et à la diffusion de documents scientifiques de niveau recherche, publiés ou non, émanant des établissements d'enseignement et de recherche français ou étrangers, des laboratoires publics ou privés. 


\title{
Fault Detection and Identification relying on Set-Membership Identifiability
}

\author{
Carine Jauberthie ${ }^{1,2}$, Nathalie Verdière ${ }^{3}$, Louise Travé-Massuyès ${ }^{1,4}$ \\ ${ }^{1}$ CNRS, LAAS, 7 avenue du Colonel Roche, F-31400 Toulouse, France \\ ${ }^{2}$ Université de Toulouse, UPS, LAAS, F-31400 Toulouse, France \\ ${ }^{3}$ Université du Havre; 25 rue Philippe Lebon; BP 540; 76058 Le Havre Cedex, France (e-mail: verdiern@univ-lehavre.fr) \\ ${ }^{4}$ Université de Toulouse, LAAS, F-31400 Toulouse, France \\ (Corresponding author: cjaubert@laas.fr, Tel: +33561336943, louise@laas.fr).
}

\begin{abstract}
Identifiability is the property that a mathematical model must satisfy to guarantee an unambiguous mapping between its parameters and the output trajectories. It is of prime importance when parameters must be estimated from experimental data representing inputoutput behavior and clearly when parameter estimation is used for fault detection and identification. Definitions of identifiability and methods for checking this property for linear and nonlinear systems are now well established and, interestingly, some scarce works $([8,16])$ have provided identifiability definitions and numerical tests in a bounded-error context. This paper resumes and better formalizes the two complementary definitions of set-membership identifiability and $\mu$-set-membership identifiability of [16] and presents a method applicable to nonlinear systems for checking them. This method is based on differential algebra and makes use of relations linking the observations, the inputs and the unknown parameters of the system. Using these results, a method for fault detection and identification is proposed. The relations mentioned above are used to estimate the uncertain parameters of the model. By building the parameter estimation scheme on the analysis of identifiability, the solution set is guaranteed to reduce to one connected set, avoiding this way the pessimism of classical set-membership estimation methods. Fault detection and identification are performed at once by checking the estimated values against the parameter nominal ranges. The method is illustrated with an example describing the capacity of a macrophage mannose receptor to endocytose a specific soluble macromolecule.

This work is a extended version of a conference paper with the same title, that appeared in the proceedings of the 8th IFAC Symposium SAFEPROCESS, August 29-31, 2012.
\end{abstract}

Keywords: Fault detection and identification; Identifiability; Uncertain dynamic systems; Nonlinear models; Bounded disturbances; Bounded noise; Parameter estimation

\section{Introduction}

Fault detection and identification via parameter estimation relies on the principle that possible faults in the monitored system can be associated with specific parameters of the mathematical model of the system given in the form of an input-output relation $y(t)=$ $g(u(t), e(t), \theta, x(t))$, where $y(t)$ represents the output vector, $u(t)$ the input vector, and $x(t)$ the state variables which are partially measurable. $\theta$ represents the non measurable parameters which are likely to change on the occurrence of a fault, and $e(t)$ the modeling error and/or noise terms affecting the process.

Identifiability is the property that the mathematical model must satisfy to guarantee an unambiguous mapping between its parameters $\theta$ and the output trajectories $y(t)$. It is of prime importance when parameters are to be estimated from experimental data representing input-output behavior and clearly when parameter estimation is used for fault detection and identification. If the model is not identifiable, it may as well be that faulty parameters and non faulty parameters are not discriminable through the estimation scheme.

Parameter estimation methods are generally cast in a stochastic framework in which uncertainty is taken into account through appropriate assumptions about noise and model error probability distributions. However, some sources of uncertainty are not well-suited to the stochastic uncertainty assumption and are better modeled as bounded uncertainty. This is typically the case for modeling tolerances on the parameter values, for which the manufacturer provides low and high bounds corresponding to the inherent variability of technological processes. Set-membership (SM) models naturally cope with this type of uncertainties and with model errors and noises assumed to be bounded but otherwise unknown.

SM methods have been the focus of a growing inter- 
est in the last decade and they have been applied to many tasks $([3,18,20])$. The literature on this topic shows interesting progress, for example, [34]. It confirms that SM estimation is an interesting alternative to stochastic estimation methods. Advantageously, SM parameter estimation methods are able to squarely reject models that are not consistent with data and error bounds. SM estimation can be based on interval analysis that was introduced by [30] and several algorithms have been proposed, like in $[17,18,21,35]$. Other approaches dedicated to linear models include ellipsoid shaped methods [15, 24, 29], parallelotopes and zonotopes [2].

Surprisingly, the interest for SM estimation methods has not been underpinned by investigations about identifiability and only two works can be mentioned. The pioneering paper by [8] outlines that interval based methods and interval constraint propagation can be used to test for a new definition of global identifiability. In contrast to structural global identifiability [32], the new property no longer allows for the existence of atypical regions in the domain of interest. This is actually a byproduct of using interval methods for testing it. But in this work, what is really an interpretation of identifiability in the SM context is only presented as a practical condition. Indeed, instead of imposing parameters corresponding to a given input-output trajectory to be strictly different, they are allowed to be distant by a given $\varepsilon$, which provides a stopping condition to the numerical method. It is only recently - ten years later that [16] formalized both the above property and test by introducing two complementary definitions for the identifiability of error-bounded uncertain models, namely set-membership identifiability (SM identifiability) and $\mu$-set-membership identifiability ( $\mu$-SM identifiability). The first one is conceptual whereas an instance of the second, called $\varepsilon$-SM identifiability, can be put in correspondence with interval based parameter estimation methods and the specified stopping condition precision threshold $\varepsilon$.

One of the benefits of SM identifiability is that it bypasses standard identifiability and allows one to give (set) estimates of parameters that are unidentifiable in the classical sense (see [40] for a good survey of classical definitions). SM identifiability indeed guarantees that there exists a mapping of the parameter space into connected subsets so that every subset can be associated with a distinguishable output behavior.

In this paper, a more formal characterization than the one in [16] is proposed for $\mu$-SM identifiability, based on the topological concept of contraction mapping [31]. This definition nicely captures the intuition that a parameter set that is associated with distinguishable be- havior may be reduced to some extent, hence the contraction, and still retain the same property. If the set can be contracted as small as desired, $\mu$-SM identifiability meets classical identifiability. Otherwise comes the concept of $\varepsilon$-SM identifiability.

Besides, a differential algebra based method for checking SM identifiability and $\mu$-SM identifiability is proposed. This method makes use of relations linking the observations, the inputs and the unknown parameters of the system. Building on this method, fault detection and identification are achieved via parameter estimation. The relations mentioned above are used to estimate the parameters of the model in a set-membership framework, through an analytic solution. The estimated value sets are then checked against the parameter nominal ranges. The identification of the fault(s) comes as a byproduct of this detection test.

The paper is organized as follows. Section 2 provides the formal description of the systems that are analyzed and of the problem. Section 3 presents the two definitions of SM identifiability. The differential algebra based method to analyze SM identifiability is given in Section 4. It is taken as a basis for parameter estimation in Section 5, and the approach is illustrated with an example describing the capacity of a macrophage mannose receptor to endocytose a specific soluble macromolecule. Finally, some conclusions are outlined in Section 6.

\section{Problem formulation}

The problem considered in this paper is the one of detecting and identifying faults that may occur on a (controlled or uncontrolled) system with bounded uncertain parameters represented by a SM model of the following form:

$$
\Gamma_{1}^{P}=\left\{\begin{array}{l}
\dot{x}(t, p)=f(x(t, p), u(t), p) \\
y(t, p)=h(x(t, p), p) \\
x\left(t_{0}, p\right)=x_{0} \in X_{0} \\
p \in P \subset \mathcal{U}_{\mathcal{P}}, t_{0} \leq t \leq T
\end{array}\right.
$$

where:

- $x(t, p) \in \mathbb{R}^{n}$ and $y(t, p) \in \mathbb{R}^{m}$ denote the state variables and the outputs at time $t$ respectively,

- $u(t) \in \mathbb{R}^{r}$ is the input vector at time $t$,

- the initial conditions $x_{0}$, if any, are supposed to belong to a bounded set $X_{0}$,

- the functions $f$ and $h$ are real and analytic on $M$ (in particular, they are infinitely differentiable), where 
$M$ is an open set of $\mathbb{R}^{n}$ such that $x(t, p) \in M$ for every $t \in\left[t_{0}, T\right]$ and $p \in P$. T is a finite or infinite time bound,

- the vector of parameters $p$ belongs to a connected set of parameters $P$. $P$ is supposed to belong to $\mathcal{U}_{\mathcal{P}}$ where $\mathcal{U}_{\mathcal{P}}$ is an a priori known set of admissible parameters, $\mathcal{U}_{\mathcal{P}}$ is either included in $\mathbb{R}^{p}$ or equal to $\mathbb{R}^{p}$.

In this paper, fault detection is approached via parameter estimation and relies on the identifiability assessment of the system as defined in section 3. It uses some relations obtained during the identifiability analysis process presented in section 4.2.

\section{Set-membership identifiability}

This section proposes a formulation of the SM identifiability problem for the class of systems formalized by (1). It resumes the definitions proposed in [16] and proposes a more formal characterization than the one in [16] for $\mu$-SM identifiability.

\subsection{Definitions}

Two definitions of global SM identifiability are provided, as well as their local counterpart. The first one is a conceptual definition, whereas the second one, relying on the definition of a contraction mapping $\mu$, can be put in correspondence with operational set-membership estimation methods. In these definitions, $Y(P, u)$ (respectively $Y(P)$ ) denotes the set of output trajectories, solution of $\Gamma_{1}^{P}$ with the input $u$ (resp. when $u=0$ ). The two following definitions apply to controlled systems, but they can be stated similarly for uncontrolled systems.

Definition 3.1. The nonempty bounded connected set $P^{*} \subseteq \mathcal{U}_{\mathcal{P}}$ is globally $S M$ identifiable if there exists an input $u$ such that $Y\left(P^{*}, u\right) \neq \emptyset$ and $Y\left(P^{*}, u\right) \cap Y(\bar{P}, u) \neq \emptyset, \bar{P} \subseteq \mathcal{U}_{\mathcal{P}} \Longrightarrow P^{*} \cap \bar{P} \neq \emptyset$.

Under the conditions of definition (3.1), we may equivalently say that the model $\Gamma_{1}^{P}$ given by (1) is globally SM identifiable with respect to $P^{*}$.

Let us now consider a nonempty bounded connected set $\Pi$ of $\mathbb{R}^{p}$ and $d$ a classical metric on $\mathbb{R}^{p}$ [7], [31]. On the metric space $(\Pi, d)$, let $\mu$ be a continuous map from $\Pi$ to $\Pi$. $\mu$ is a contraction if there is a nonnegative number $k<1$ such that for all $\pi_{1}, \pi_{2}$ in П, $d\left(\mu\left(\pi_{1}\right), \mu\left(\pi_{2}\right)\right)<k d\left(\pi_{1}, \pi_{2}\right)$ [31]. Let us also define the diameter of $\Pi$ by the least upper bound of $\left\{d\left(\pi_{1}, \pi_{2}\right), \pi_{1}, \pi_{2} \in \Pi\right\}$.
In the following definition, the set $P^{*}$ is supposed to be a bounded connected set and $\mu$ is a contraction from $P^{*}$ to $P^{*}$.

Definition 3.2. The nonempty bounded connected set $P^{*} \subseteq \mathcal{U}_{\mathcal{P}}$ is globally $\mu$-SM identifiable if, for all contractions $\mu$ from $P^{*}$ to $P^{*}, \mu\left(P^{*}\right)$ is globally SM identifiable.

Under the conditions of definition (3.2), we may equivalently say that the model $\Gamma_{1}^{P}$ given by (1) is globally $\mu$-SM identifiable with respect to $P^{*}$.

Definition 3.2 differs from definition 3.1 in the sense that the set $P^{*}$ may be reduced as small as desired by the contraction $\mu$ while still retaining the property of SM identifiability. This is true by Banach fixed-point theorem, which implies that the diameter of $\mu\left(P^{*}\right)$ tends to zero [31]. In this case, $\mu$-SM identifiability meets classical identifiability and, interestingly, it means that classical identifiability holds for any $p \in P^{*}$. In the opposite case, i.e. if the diameter of $\mu\left(P^{*}\right)$ cannot be lower than $\varepsilon$ without eventually loosing SM identifiability, we refer to $\varepsilon$-SM identifiability. This definition will be shown to have practical importance in section 3.3.

The definition of $\mu$-SM identifiability hence subsumes classical identifiability, while leading to the concept of $\varepsilon$-SM identifiability, which is an extension to the SM framework.

To account for possible singularities in $\mathcal{U}_{\mathcal{P}}, \mu$-SM identifiability can be generically extended into structural $\mu$-SM identifiability, which means that the model set $\Gamma_{1}^{P}$ is $\mu$-SM identifiable with respect to $P^{*} \subset \mathcal{U}_{\mathcal{P}}$ except for a subset of points of zero measure in $\mathcal{U}_{\mathcal{P}}$. Let us notice that defining the structural counterpart of SM identifiability, as given by definition 3.1, is not relevant because in this definition, $P^{*}$ cannot be of zero measure. The same is true for $\varepsilon$-SM identifiability as explained in [8].

Proposition 3.1. Global $\mu$-SM identifiability with respect to $P^{*}$ implies global SM identifiability with respect to $P^{*}$ but the inverse is not true.

Proof - The implication is obvious. The fact that the inverse implication is not true is proved with a counterexample. Let us consider the following uncertain model in which $\omega$ is an unknown parameter allowed to take an interval value:

$$
\left\{\begin{array}{l}
\dot{x}=x+t \cos (\omega) \\
x(0)=x_{0} \in X_{0}
\end{array}\right.
$$

Its solution is $x(t)=x_{0} e^{t}+\left(-1-t+e^{t}\right) \cos (\omega)$. An admissible set for $\omega$ is taken as $\mathcal{U}_{\mathcal{P}}=[0,2 \pi]$. If 
$\omega \in P^{*}=\left[\frac{\pi}{2}, \frac{3 \pi}{2}\right]$, the model is globally SM identifiable but not $\mu$-SM identifiable. Indeed, no trajectory arising from the systems whose parameter $\omega$ is in $P^{*}$ is identical to a trajectory arising from the complementary set of $P^{*}$ in $\mathcal{U}_{\mathcal{P}}$, hence SM identifiability of $P^{*}$. However, if the diameter of $P^{*}$ is smaller, there may exist two disjoint subsets of $P^{*}$, namely $P_{1}^{*}$ and $P_{2}^{*}$, which result in common trajectories. This is the case, for example, of $P_{1}^{*}=\left[\pi, \frac{3 \pi}{2}\right]$ and $P_{2}^{*}=\left[\frac{\pi}{2}, \frac{3 \pi}{4}\right]$. Consequently, $P^{*}$ cannot be contracted as small as desired, and is not hence $\mu$-SM identifiable.

Local definitions of $(\mu-)$ SM identifiability can be given by considering an open neighborhood $W$ of $P^{*}$ in which $\Gamma_{1}^{P}$ is globally ( $\mu$-)SM identifiable with respect to $P^{*}$ with $\mathcal{U}_{\mathcal{P}}$ restricted to $W$.

If the model (1) is neither globally $(\mu-) \mathrm{SM}$ identifiable nor locally $(\mu-) \mathrm{SM}$ identifiable, it is said non $(\mu$ )SM identifiable.

In the case of uncontrolled models, similar definitions can be considered without input.

\section{2. $\mu$-SM-identifiability and parameter estimation}

Parameter estimation algorithms consist in most of the cases in comparing the observed signal with the system output obtained with candidate (interval) parameter vectors. The observed signal is generally uncertain and can be defined within bounds distant from a given $\lambda$. The parameters whose trajectories fall within these bounds are admissible, whereas the others are not. $\mu$ SM identifiability with respect to $P^{*}$ implies that the trajectories arising from the two sets, namely $P^{*}$ and its complementary set, are not identical. But this is not enough to guaranty that, if the trajectories arising from $P^{*}$ fall within the bounds, none of the trajectories of the complementary set does.

Using Gronwall lemma and assuming that the functions $f$ and $h$ defining model (1) are Lipschitz, it has been proved in [39] that $y(t, p)$ is $M$-Lipschitz with respect to the second variable, i.e. the parameter vector. The constant $M$ has been defined explicitly so that the conditions under which the output trajectories $y(t, p)$ are distant by a given $\lambda$ can be determined.

This condition together with $\mu$-SM identifiability with respect to $P^{*}$ not only imply that the trajectories arising from a set $P^{*}$ and its complementary set are different but also that there exist at least a time point for which they are distant by more than $\lambda$.

In other words, one can determine the minimal distance between two parameter vectors for which it is possible to distinguish numerically the trajectories by $\lambda$.
Moreover, once the parameter vector precision is given, the time point for which the distance between two trajectories, in $P^{*}$ and its complementary set, is above $\lambda$ can be determined.

\subsection{Correspondence with operational interval based parameter estimation}

This section first provides some concepts related to the manipulation of sets, then discusses interval based set inversion, exemplified by the algorithm SIVIA (Set Inversion Via Interval Analysis), as a framework in which the parameter estimation problem can be cast [18]. An interpretation of $\varepsilon$-SM identifiability is then exhibited and shown to be a formalization of the interval based test proposed in [8] in the framework of Interval Constraint Propagation (ICP). $\varepsilon$-SM identifiability is then shown to generalize classical identifiability to sets whose dimension can be controlled.

\subsubsection{Interval set inversion}

When manipulating sets of values, it is important to be able to check whether one set is included in another set or not. Given two subsets $\mathbb{S}_{1}$ and $\mathbb{S}_{2}$ of $\mathbb{R}^{n}$, one wants to test whether $\mathbb{S}_{1}$ is included in $\mathbb{S}_{2}$ or not. This test, known as the inclusion test is used to prove that all points in a given set satisfy a given property or to prove that none of them does.

Conversely, if two sets intersect, their intersection inherits the properties of the two sets. It is hence often desirable to reduce a set to its intersection with respect to another set, which is obtained through contraction. The contraction ${ }^{1}$ of $\mathbb{S}_{1}$ with respect to $\mathbb{S}_{2}$ is a smaller set $s$ such that $\mathbb{S}_{1} \cap \mathbb{S}_{2}=s \cap \mathbb{S}_{2}$. If $\mathbb{S}_{2}$ is the feasibility set of a problem and $s$ turns out to be empty, then the set $\mathbb{S}_{1}$ does not contain the solution.

Interval analysis makes use of specific sets, also known as boxes. A real interval is a closed and connected subset of $\mathbb{R}$ denoted $[\underline{x}, \bar{x}]=\{x \in \mathbb{R}, \mid \underline{x} \leq x \leq$ $\bar{x}\}$. A box is an interval vector $[\underline{X}, \bar{X}]$, that is a vector with interval components.

In SIVIA, inclusion and contraction are used to test if a box can or cannot be removed from the solution set. When no conclusion can be drawn, the box is bisected and each of the sub-boxes can be tested in turn in a branch-and-bound manner. The same principles are used in ICP.

\footnotetext{
${ }^{1}$ Although the intuition behind contraction as used here and contraction as used in section 3.1 is similar, the two concepts are not the same. We use the same term because it is used as so by the interval analysis community.
} 
Consider the problem of determining the solution set for the unknown quantity $u$ defined by:

$$
\begin{aligned}
S & =\{u \in U \mid \Phi(u) \in[y]\}, \\
& =\Phi^{-1}([y]) \cap U,
\end{aligned}
$$

where $[y]$ is known a priori, $U$ is an a priori search set for $u$ and $\Phi$ a nonlinear function not necessarily invertible in the classical sense. (3) involves computing the reciprocal image of $\Phi$. This can be solved using the algorithm SIVIA, which recursively explores all the search space without loosing any solution. SIVIA delivers a guaranteed enclosure of the solution set $S$ as follows:

$$
\underline{S} \subseteq S \subseteq \bar{S}
$$

The inner enclosure $\underline{S}$ is composed of the boxes that have been proved feasible. To prove that a box $[u]$ is feasible it is sufficient to prove that $\Phi([u]) \subseteq[y]$. Reversely, if it can be proved that $\Phi([u]) \cap[y]=\emptyset$, then the box $[u]$ is unfeasible. Otherwise, no conclusion can be reached and the box $[u]$ is said undetermined. The latter is then bisected in two sub-boxes that are tested until their size reaches a user-specified precision threshold $\varepsilon>0$. Such a termination criterion ensures that SIVIA terminates after a finite number of iterations.

In the above solving schema, the number of bisections to be performed is generally prohibitive. Hence, recent algorithms take advantage of constraint propagation techniques to reduce the width of the boxes to be tested [12], [13], [42]. In this context, the inclusion relations and the model equations can be interpreted as constraints. A Constraint Satisfaction Problem $(C S P)$ can be formulated as below.

A Constraint Satisfaction Problem $H=(\mathcal{X}, \mathcal{D}, \mathcal{C})$ is defined by:

- a set of variables $\mathcal{X}=\left\{x_{1}, \ldots, x_{n}\right\}$,

- a set of nonempty domains $\mathcal{D}=\left\{D_{1}, \ldots, D_{n}\right\}$ where $D_{i}$ is the domain associated to the variable $x_{i}$,

- a set of constraints $\mathcal{C}=\left\{C_{1}, \ldots, C_{m}\right\}$, so that the associated variables to each constraint are defined in $\mathcal{X}$.

Given a $C S P$ and an initial box $\mathbb{S}_{1}$ (which may result from a bisection operation as explained above), different types of so-called contractors can be used [17][9] to contract $\mathbb{S}_{1}$ into a set $s$ that satisfies the constraints. Among the most well-known is the forward-backward contractor [5] which is based on constraint propagation and consists in contracting the domain of the CSP by taking into account iteratively each constraint of the set $\left\{C_{1}, \ldots, C_{m}\right\}$.

\subsubsection{Interpretation of $\varepsilon-S M$ identifiability}

SM identifiability does not provide the means to control the set of interest $P^{*}$, i.e. the parameter solution set. This points out the practical interest of $\mu$-SM identifiability which is defined through a contraction mapping $\mu($.$) that allows one to control the diameter of P^{*}$. In particular, $\varepsilon$-SM identifiability means that the diameter of $P^{*}$ cannot be lower than $\varepsilon$ without eventually loosing SM identifiability. The diameter of $P^{*}$ can hence be put in correspondance with the user-specified precision threshold of SIVIA. Consequently, $\varepsilon$-SM identifiability provides the means to guarantee that the estimate provided by the set inversion algorithm when the precision threshold is taken equal to $\varepsilon$ consists of a connected set.

$\varepsilon$-SM identifiability is actually a formalization of the ICP numerical test proposed by [8] to check global identifiability in a domain. Instead of imposing parameters corresponding to a given input-output trajectory to be strictly different, [8] allows them to be distant by a given $\varepsilon$, which provides a stopping condition to the ICP numerical method.

Ultimately, $\mu$-SM identifiability subsumes classical identifiability and SM identifiability as it provides the means to control the set $P^{*}$ thanks to the contraction mapping $\mu($.$) . When the diameter of P^{*}$ tends to 0 , $\mu$-SM identifiability comes back to classical identifiability. Interestingly, $\mu$-SM identifiability with respect to $P^{*}$ means that classical identifiability holds for any $p \in P^{*}$, which provides a way to test classical identifiability for a set of parameters as a whole as shown in section 4 . When this diameter is necessarily higher or equal to $\varepsilon$, it results in $\varepsilon$-SM identifiability.

\section{Analysis of set-membership identifiability}

In the literature, different approaches have been proposed for studying global identifiability of nonlinear systems, for example, the revisited Taylor Series approach of [33], those based on the local state isomorphism theorem, [10], [11], [19], [41], or those based on differential algebra, [4], [28], [36], [38]. Most of them can be adapted in order to test global ( $\mu$-)SM identifiability. In [39], the authors propose two methods for testing the global $(\mu$-)SM identifiability: the first one is based on the Taylor Series approach, the second one on differential algebra. The latter is used in this paper because, as it will be seen in section 5 , it permits to deduce 
a numerical procedure for SM fault detection. The notion of partial injectivity of [23] is needed and is recalled in the first subsection.

\subsection{Partial injectivity}

Definition 4.1. Consider a function $f: \mathcal{A} \rightarrow \mathcal{B}$ and any set $\mathcal{A}_{1} \subseteq \mathcal{A}$. The function $f$ is said to be a partial injection of $\mathcal{A}_{1}$ over $\mathcal{A}$, noted $\left(\mathcal{A}_{1}, \mathcal{A}\right)$-injective, if $\forall a_{1} \in \mathcal{A}_{1}, \forall a \in \mathcal{A}$,

$$
a_{1} \neq a \Rightarrow f\left(a_{1}\right) \neq f(a) .
$$

$f$ is said to be $\mathcal{A}$-injective if it is $(\mathcal{A}, \mathcal{A})$-injective.

In [23], an algorithm based on interval analysis for testing the injectivity of a given differentiable function is presented and a solver called ITVIA (Injectivity Test Via Interval Analysis) implemented in C++ is mentioned.

\subsection{Testing global ( $\mu$-)SM identifiability by Differential Algebra}

The proposed approach is an extension of [16] and is based on [38]. [14] has proved that, choosing the appropriate elimination order $\{p\}<\{y, u\}<\{x\}$ (which consists in eliminating unobservable state variables), the differential algebra approach [22] allows one to obtain relations between inputs, outputs and parameters. These relations can be expressed as:

$$
\begin{aligned}
R_{i}(y, u, p)= & \theta_{0}^{i}(y, u) \\
& +\sum_{k=1}^{n_{i}} \theta_{k}^{i}(p) m_{k}^{i}(y, u), i=1, \ldots, m,
\end{aligned}
$$

where $\left(\theta_{k}^{i}\right)_{1 \leq k \leq n_{i}}$ are rational in $p, \theta_{u}^{i} \neq \theta_{v}^{i}(u \neq v)$, $\left(m_{k}^{i}(y, u)\right)_{1 \leq k \leq n_{i}}$ are differential polynomials with respect to $y$ and $u$ and $\theta_{0}^{i} \neq 0$.

The size of the system is the number of observations. For simplicity, we assume that $i=1$, i.e. there is only one output variable and $n=n_{1}, m_{k}(y, u)=m_{k}^{1}(y, u)$, $\theta_{k}=\theta_{k}^{1}$. The higher order derivative of $y$ in (5) is noted $l$.

The following theorem provides necessary and sufficient conditions for global SM identifiability or $\mu$-SM identifiability.

Theorem 1. Assume that the functional determinant $\triangle R(y, u)=\operatorname{det}\left(m_{k}(y, u), k=1, \ldots, n\right)$, is not in the ideal $\mathcal{I}_{p}^{0}$ obtained after eliminating state variables. Consider $P^{*}$ a subset of $\mathcal{U}_{\mathcal{P}}$ for which the function $\phi$ defined by

$$
\begin{aligned}
\phi: p=\left(p_{1}, \ldots, p_{p}\right) \mapsto \quad & \left(\theta_{1}(p), \ldots, \theta_{n}(p), y\left(t_{0}^{+}, p\right),\right. \\
\ldots, & \left.y^{(l-1)}\left(t_{0}^{+}, p\right)\right)
\end{aligned}
$$

verifies:

$$
\forall p^{*} \in P^{*}, \forall \bar{p} \notin P^{*}, \phi\left(p^{*}\right) \neq \phi(\bar{p}) .
$$

Then the model $\Gamma_{1}^{P}$ is globally SM identifiable with respect to $P^{*}$.

If the model $\Gamma_{1}^{P}$ is globally SM identifiable with respect to $P^{*}$ and $\phi$ is $\left(P^{*}, \mathcal{U}_{\mathcal{P}}\right)$-injective then the model is $\mu$ $S M$ identifiable with respect to $P^{*}$.

In the two cases, if the coefficient of $y^{(l)}$ in (5) is not equal to 0 at $t_{0}$, then the reciprocal is valid.

Proof - Sufficiency Consider $P^{*}$ verifying the hypothesis of the theorem. Suppose there exists an input $u^{*}$ such that $Y\left(P^{*}, u^{*}\right) \neq \emptyset$ and $y^{*} \in Y\left(P^{*}, u^{*}\right) \cap$ $Y\left(\bar{P}, u^{*}\right)$ for a cartesian product of intervals $\bar{P} \in \mathcal{U}_{\mathcal{P}}$. Thus, there exists $p^{*} \in P^{*}, \bar{p} \in \bar{P}$ such that $y^{*}=$ $y\left(., p^{*}\right)=y(., \bar{p})$ and $R\left(y^{*}, u^{*}, p^{*}\right)=R\left(y^{*}, u^{*}, \bar{p}\right)$.

Denote $Q\left(y^{*}, u^{*}\right)=R\left(y^{*}, u^{*}, p^{*}\right)-R\left(y^{*}, u^{*}, \bar{p}\right)$. Since $\operatorname{det}(Q)\left(y^{*}, u^{*}\right)=\operatorname{det}\left(m_{k}\left(y^{*}, u^{*}\right), k=0, \ldots, n\right)=$ $\triangle R\left(y^{*}, u^{*}\right)$ is not equal to zero, $\theta_{k}\left(p^{*}\right)=\theta_{k}(\bar{p})$ for $k=1, \ldots, n$. Furthermore, recall that $y\left(., p^{*}\right)=y(., \bar{p})$ in particular $y^{(j)}\left(t_{0}, p^{*}\right)=y^{(j)}\left(t_{0}, \bar{p}\right)$ for $j=0, \ldots, l-$ 1. Since the function $\phi$ is supposed to verify condition (6), one gets $\bar{p} \in P^{*}$ that is $P^{*} \cap \bar{P} \neq \emptyset$.

If $\phi$ is $\left(P^{*}, \mathcal{U}_{\mathcal{P}}\right)$-injective then, $\phi$ is in particular injective on $P^{*}$ and we always have $p^{*}=\bar{p}$, that is $P^{*} \cap \bar{P} \neq \emptyset$.

Necessity Let's prove the contrapositive. Suppose there exists $\bar{P}, P^{*} \cap \bar{P}=\emptyset$ containing $\bar{p}$ such that $\phi\left(p^{*}\right)=\phi(\bar{p})$ for a certain $p^{*} \in P^{*}$. Since the coefficient of $y^{(l)}$ in (5) is not equal to 0 at $t_{0}$ and the differential polynomials $\left(m_{k}\right)_{k=1, \ldots, n}$ have a degree 1 in $y^{(l)}$ ([14]), any time derivative $y^{(r)}\left(t_{0}^{+}, p^{*}\right), r \geq l$ can be rewritten as a function of $y^{(l-1)}\left(t_{0}^{+}, p^{*}\right), \ldots, y\left(t_{0}^{+}, p^{*}\right), \theta_{1}\left(p^{*}\right), \ldots, \theta_{n}\left(p^{*}\right)$. According to the hypothesis, the coefficients of $y\left(t, p^{*}\right)$ in the Taylor expansion are the same as those of $y(t, \bar{p})$. Thus, $y^{*}:=y\left(t, p^{*}\right)=y(t, \bar{p})$ and $y^{*} \in Y\left(P^{*}, u\right) \cap$ $Y(\bar{P}, u)$. Consequently, the model is not globally SM identifiable for $P^{*}$.

Example: Consider the uncertain model:

$$
\left\{\begin{array}{l}
\dot{x}_{1}=x_{1}^{2}+\left(1-p_{2}\right) x_{2}, \\
\dot{x}_{2}=\sin \left(p_{1}\right) x_{1}, \\
x_{1}(0)=\left(p_{1}+2\left(1-p_{2}\right) \cos \left(p_{1}\right)\right), \\
x_{2}(0)=0, \\
y=x_{1},
\end{array}\right.
$$

where $\left(p_{1}, p_{2}\right) \in P^{*}=[-1,4] \times[0,1 / 10]$.

We want to know whether the model is globally $\mu$-SM identifiable with respect to $P^{*}$. By setting $c_{1}=\sin \left(p_{1}\right)$ 
and with the elimination order $\left\{c_{1}, p_{2}\right\}<\{y\}<$ $\left\{x_{1}, x_{2}\right\}$, the package diffalg of Maple gives the following input-output polynomial:

$$
R(y, u)=\ddot{y}-2 \dot{y} y-\left(1-p_{2}\right) c_{1} y .
$$

In that case, the functional determinant is reduced to $\triangle R(y, u)=y$ and in using the function belong_to of the package diffalg of Maple [6], we verify that it is not in $\mathcal{I}_{p}^{0}$.

In order to consider the initial condition, the function $\phi$ : $\left(p_{1}, p_{2}\right) \rightarrow\left(\left(1-p_{2}\right) \sin \left(p_{1}\right),\left(p_{1}+2\left(1-p_{2}\right) \cos \left(p_{1}\right)\right)\right)$ has to be studied. Notice that the coefficient of $\ddot{y}$ in (5) is not equal to 0 at 0 . The solver ITVIA ([23]) allows one to obtain the partition of the box $P^{*}$ on which the function $\Phi$ is partially injective and $\Phi$ can be proved to be not injective over $P^{*}$. Thus the model is not $\mu$-SM identifiable with respect to $P^{*}$.

\section{Fault detection and identification}

\subsection{Problem setting}

SM fault detection may use state estimation or parameters estimation. When the model is nonlinear like (1), the sets to be characterized (state or parameter values) may be nonconvex and may even consist of several disconnected sets. In the latter case, interval analysis encloses such sets in the convex hull and the usual drawback is to obtain very conservative interval solution vectors, which result in missing alarms. Recent methods such as [1] or the one proposed in this paper should provide significant improvements in this direction.

In this work, the same analysis is used for identifiability checking and for estimating the parameters. Advantageously, after analyzing identifiability, we can guarantee that the solution set for the system (1) reduces to one connected set.

In this section, a numerical method deduced from section 4.2 is proposed to estimate the unknown constant parameters of a non linear system like (1).

The output $y$ is supposed to be disturbed by a bounded additive noise $\eta, \eta(t) \in[\eta(t)]$ and the parameter vector $p$ belongs to $P$ where $P$ is an interval vector. The polynom (5) can be used to estimate the interval vector $P$. Consider $\Theta_{k}$ the associated expression of $\theta_{k}$ defined in the polynom (5), where $p$ is substituted by $P$. $\Theta_{k}(P)$ is a connected set for all connected $P$ since it involves sum, difference and product of connected sets. Suppose that the observations are done at discrete times $t_{j}, 0 \leq j \leq M$ and they are noted $y_{j}=y\left(t_{j}\right)$.
Then, the following system whose interval vector $\left(\Theta_{k}(P)\right)_{1 \leq k \leq n}$ is unknown can be deduced:

$$
\begin{aligned}
& \forall j=0, \ldots, M, \\
& 0 \in m_{0}\left(y_{j}, u_{j}\right)+\sum_{k=1}^{n} \Theta_{k}(P) m_{k}\left(y_{j}, u_{j}\right) .
\end{aligned}
$$

Notice that (8) is linear with respect to $\left\{\Theta_{1}(P), \ldots, \Theta_{n}(P)\right\}$. Solving the previous system comes back to solving $0 \in[A][x]-[b]$ or $[A][x]=[b]$ where $[A]_{j}=\left(\left[m_{k}\left(y_{j}, u_{j}\right)\right]\right)_{k=1, \ldots, n}$ is the $j^{\text {th }}$ line of the interval matrix $[A]$ and $[b]_{j}=-\left[m_{0}\left(y_{j}, u_{j}\right)\right]$ is the $j^{\text {th }}$ line of the interval vector $[b]$.

Finding a solution for (8) requires an evaluation of a finite number of measurement derivatives. In this work, these derivatives are estimated by using Higher Order Sliding Mode (HOSM) differentiators developped in [25], [27].

\subsection{Derivative estimation}

In the works [25], [26], [27] concerning HOSM differentiators, the input signal $y(t)$ to be derivated is considered as a function defined on $[0,+\infty[$. The signal $y(t)$ is supposed to consist of a bounded Lebesguemeasurable noise (bounded by a positive constant $\alpha$ ) with unknown features and an unknown base signal $y_{0}(t)$ with the $m^{t h}$ derivative having a known Lipschitz constant $C>0$. The successive derivatives of a signal $y(t)$ are estimated by $z_{0}(t), z_{1}(t), \ldots, z_{m}(t)$ as described below:

$$
\left\{\begin{aligned}
\dot{z}_{0}= & v_{0}, \\
v_{0}= & -\lambda_{0}\left|z_{0}-y\right|^{\frac{m}{(m+1)}} \operatorname{sign}\left(z_{0}-y\right)+z_{1}, \\
\dot{z_{1}}= & v_{1}, \\
v_{1}= & -\lambda_{1}\left|z_{1}-v_{0}\right|^{\frac{(m-1)}{m}} \operatorname{sign}\left(z_{1}-v_{0}\right)+z_{2}, \\
\vdots & \\
z_{m-1}= & v_{m-1}, \\
v_{m-1}= & -\lambda_{m-1}\left|z_{m-1}-v_{m-2}\right|^{\frac{1}{2}} \operatorname{sign}\left(z_{m-1}-v_{m-2}\right) \\
& +z_{m}, \\
\dot{z_{m}=} & -\lambda_{m} \operatorname{sign}\left(z_{m}-v_{m-1}\right),
\end{aligned}\right.
$$

where $\lambda_{j} \in \mathbb{R}, j=0, \ldots, m$ represent the differentiator parameters. Generally, these parameters are chosen experimentally (for more details, see [25], [26]).

It has been proved in [26] that the best estimate accuracy of the $k^{t h}$ derivative is proportional to:

$$
a c c_{k}=\mu_{k} C^{\frac{k}{m+1}} \alpha^{\frac{m+1-k}{m+1}}, \quad k=0,1,2, \ldots m
$$

where $\mu_{k} \geq 1$ depends only on $\lambda_{j}(j=0, \ldots, m)$. In this paper, we compute an interval containing the exact $k^{t h}$ derivative of $y$. We note $y_{p}^{(k)}$ the estimate of the 
$k^{t h}$ exact derivative of $y$, then this computed interval is given by:

$$
\left[y_{p}^{(k)}-a c c_{k}, y_{p}^{(k)}+a c c_{k}\right]
$$

with $y_{p}^{(k)}=z_{k}[37]$.

\subsection{Case study}

The following example taken from [38] is considered. In [38], the proposed model is cast in a stochastic framework in which uncertainty is taken into account through appropriate assumptions about noise and model error probability distributions.

This model allows one to explore the capacity of the macrophage mannose receptor to endocytose soluble macromolecule and to quantify the different aspects of such a process:

$$
\left\{\begin{array}{l}
\dot{x}_{1}=\alpha_{1}\left(x_{2}-x_{1}\right)-\frac{V_{m} x_{1}}{1+x_{1}} \\
\dot{x}_{2}=\alpha_{2}\left(x_{1}-x_{2}\right) \\
x_{1}(0) \in[0.62,0.63], x_{2}(0)=0 \\
y=x_{1}
\end{array}\right.
$$

where $x_{1}$ (resp. $x_{2}$ ) is the enzyme concentration outside (resp. inside) the macrophage and $p=\left(\alpha_{1}, V_{m}, \alpha_{2}\right)$ are the unknown parameters which have to be identified. The parameter $\alpha_{1}$ is the rate constant of the transfer from Compartment 1 (or the central compartment), practically plasma, to Compartment 2 (or the peripheral compartment), which represents the part of the extravascular extracellular fluid accessible. Furthermore, $\alpha_{2}$ is the rate constant of the transfer from Compartment 2 to Compartment 1.

This model can be easily proved to be globally $\mu$-SM identifiable with respect to $\left(\mathbb{R}^{+}\right)^{3}$. The numerical study has been conducted in simulation in Matlab using Intlab. The simulated outputs are disturbed by a truncated gaussian noise $\eta$ such that $\eta(t) \in[-0.001,0.001]$. Thus, $y(t)=\bar{y}(t)+\eta(t)$ where $\bar{y}$ is the exact output corresponding to the exact value of parameters: $\alpha_{1}=$ $0.011, \alpha_{2}=0.02$ and $V_{m}=0.1$. The observations are supposed to be done at discrete times $\left(t_{j}\right)_{j=1, \ldots, N}$ on the interval $[0,60]$ with a sampling period equal to 1 . The polynom $R(y, u)$ is given by:

$R(y, u)=\ddot{y}(1+y)^{2}+\gamma_{1} \dot{y}(1+y)^{2}+\gamma_{2} y(1+y)+\gamma_{3} \dot{y}$,

with $\gamma_{1}=\alpha_{1}+\alpha_{2}, \gamma_{2}=\alpha_{2} . V_{m}$ and $\gamma_{3}=V_{m}$.

If we denote $y_{p}^{(1)}\left(t_{j}\right)$ (resp. $y_{p}^{(2)}\left(t_{j}\right)$ ) the estimate of $\dot{y}\left(t_{j}\right)$ (resp. $\ddot{y}\left(t_{j}\right)$ ), the obtained system which has to be solved is $[A][x]=[b]$ where $[A]_{j}=\left(\left[y_{p}^{(1)}\left(t_{j}\right)(1+\right.\right.$ $\left.\left.\left.y\left(t_{j}\right)\right)^{2}\right], \quad\left[y\left(t_{j}\right)\left(1+y\left(t_{j}\right)\right)\right], \quad\left[y_{p}^{(1)}\left(t_{j}\right)\right]\right)$ and $[b]_{j}=$ $\left[-y_{p}^{(2)}\left(t_{j}\right)\left(1+y\left(t_{j}\right)\right)^{2}\right]$.

$y_{p}^{(1)}\left(t_{j}\right)$ and $y_{p}^{(2)}\left(t_{j}\right)$ are obtained by using HOSM differentiators presented in subsection 5.2. The parameters of the HOSM differentiators are given by $\lambda_{0}=3$, $\lambda_{1}=0.2$ and $\lambda_{2}=0.1$.

Solving this system can be cast into the set inversion framework for which we used the SIVIA algorithm complented by the forward-backward propagation to contract the initial parameter box. The problem solved here is to find $[x]$ such that $0 \in[A][x]-[b]$, given initial intervals for $\gamma_{1}, \gamma_{2}$ and $\gamma_{3}$ obtained from prior knowledge.

Case of nominal behaviour: By using initial intervals given by $\gamma_{1}=\left[\begin{array}{ll}0, & 0.04\end{array}\right], \gamma_{2}=\left[\begin{array}{ll}0, & 0.003\end{array}\right]$, $\gamma_{3}=[0,0.2]$ and the bisection precision $\varepsilon=0.001$, we obtain in 14.18 seconds : $\alpha_{1}=[0,0.0401], \alpha_{2}=$ $[0,0.0437]$ and $V_{m}=[0.06875,0.13203]$,

by using the equations: $V_{m}=\gamma_{3}, \alpha_{2}=$ $\gamma_{2} / V_{m}, \alpha_{1}=\gamma_{1}-\alpha_{2}$. All these intervals contain the normal values, confirming normal behavior.

Then, by using $\gamma_{1}=\left[\begin{array}{ll}0, & 0.04\end{array}\right], \quad \gamma_{2}=$ $\left[\begin{array}{ll}0,0.003\end{array}\right], \gamma_{3}=\left[\begin{array}{ll}0, & 0.2\end{array}\right]$ and the bisection precision $\varepsilon=0.0001$, we obtain in 177.55 seconds : $\alpha_{1}=[0,0.0329], \alpha_{2}=[0.0071,0.0317]$ and $V_{m}=[0.094824,0.10527]$.

All these intervals contain the normal values.

Case of a fault on parameter $\alpha_{2}$ : In this simulation, we assume a fault on $\alpha_{2}=2$, which means that the rate of the transfer from Compartment 2 to Compartment 1 is high.

After 25.15 minutes, by using $\gamma_{1}=[0,3], \gamma_{2}=$ $[0,1], \quad \gamma_{3}=\left[\begin{array}{ll}0, & 0.2\end{array}\right]$, we obtain: $\alpha_{1}=$ $[0.0000,0.5050], \alpha_{2}=[1.1200,10.4435]$ and $V_{m}=$ $[0.0242,0.1790]$.

The real faulty value of $\alpha_{2}$ is contained in the estimated interval for $\alpha_{2}$, which allows us to detect and localize the fault. Moreover, there is no intersection between the estimated interval for $\alpha_{2}$ and the one obtained for normal behaviour.

Case of a fault on parameter $\alpha_{2}$ at $t=15$ s: Consider now the case of an abrupt change in the value of $\alpha_{2}$ during the test and let us assume a fault $\alpha_{2}=1$ at time $t=15 \mathrm{~s}$. This fault is detected in $t=0.05 \mathrm{~s}$ after its occurence.

After the detection of this fault, by using $\gamma_{1}=[0,1.05]$, $\gamma_{2}=[0,0.12], \gamma_{3}=[0,0.12]$, we obtain in 22.6 seconds $\alpha_{1}=[0,0.0238], \alpha_{2}=[0.0738,1.0500]$ and $V_{m}=[0,0.1200]$. These intervals on $\alpha_{1}$ and $V_{m}$ 
contain the normal values whereas the one obtained for $\alpha_{2}$ contains the faulty value.

Case of a fault on parameter $V_{m}$ : In this simulation, we assume a fault on $V_{m}=0.2$ at $t=27 \mathrm{~s}$. This fault is detected in $t=0.02 \mathrm{~s}$ after its occurence. Once the fault is detected, the estimation algorithm is initialized with $\gamma_{1}=[0,0.04], \gamma_{2}=[0,0.007], \gamma_{3}=[0,0.3]$, and we obtain in $33.43 \mathrm{~s}$ the intervals $\alpha_{1}=[0,0.0150]$, $\alpha_{2}=[0,0.0248]$ and $V_{m}=[0.0242,0.3]$.

The intervals on $\alpha_{1}$ and $\alpha_{2}$ contain the normal values whereas the one for $V_{m}$ contains the faulty value, hence confirming the fault.

\section{Conclusion}

This paper proposes a fault detection and identification method for bounded uncertainty nonlinear models relying on an original parameter identifiability scheme. It takes benefit of a differential algebra based method for checking SM identifiability and its operational counterpart $\mu$-SM identifiability. These notions provide a way to study different aspects of identifiability for uncertain bounded-error systems, in particular systems that represent an infinite family of nonlinear systems. By building the parameter estimation scheme on the analysis of identifiability, we guarantee that the solution set reduces to one connected set, avoiding this way the pessimism of SM methods. Identifiability is closely related to diagnosability as it provides the guaranty that two situations corresponding to different parameterized settings are distinguishable. The proposed method has been applied to an example describing the capacity of a macrophage mannose receptor to endocytose a specific soluble macromolecule. Different normal and faulty scenarios have been considered. For every scenario, the parameters have been estimated correctly with reasonable precision.

Acknowledgments: This work was supported by the French National Research Agency (ANR) in the framework of the project ANR-11-INSE-006 (MAGIC-SPS).

\section{References}

[1] O. Adrot and S. Ploix. Fault detection based on set-membership inversion. In Proceedings of SAFEPROCESS'06, pages 619624, Beijing, China, 2006

[2] T. Alamo, J. Bravo, and E. Camacho. Guaranteed state estimation by zonotopes. Automatica, 41(6):1035-1043, 2005.

[3] J. Armengol, L. Travé-Massuyès, J. Vehi, and J.Ll. de la Rosa. A survey on interval model simulators and their properties related to fault detection. Annual Reviews in Control, 24:31-39, 2000.
[4] S. Audoly, G. Bellu, L. D’Angio, M. P. Saccomani, and C. Cobelli. Global identifiability of nonlinear models of biological systems. IEEE Trans. Biomed. Eng., 48:55-65, 2001.

[5] F. Benhamou, F. Goualard, F. Granvilliers, and J.F. Puget. Revising hull and box consistency. In Proceedings of the International Conference on Logic Programming, pages 230-244, Las Cruces, New Mexico, 1999.

[6] F. Boulier, D. Lazard, F. Ollivier, and M. Petitot. Computing representations for the radicals of a finitely generated differential ideals. Technical report IT306, LIFL, University of Lille, 1997.

[7] N. Bourbaki. Elements of Mathematics. Springer-Verlag, 1989.

[8] I. Braems, L. Jaulin, M. Kieffer, and E. Walter. Guaranteed numerical alternatives to structural identiability testing. In Proceedings of the 40th IEEE CDC, pages 3122-3127, Orlando, USA, 2001.

[9] G. Chabert and L. Jaulin. Contractor programming. Artifical Intelligence, 173:1079-1100, 2009.

[10] M.J. Chapman, K.R. Godfrey, M.J. Chappell, and N.D. Evans. Structural identifiability of nonlinear systems using linear/nonlinear splitting. Int. J. Control, 76:209-216, 2003.

[11] M.J. Chappell and K.R. Godfrey. Structural identifiability of the parameters of a nonlinear batch reactor model. Math. Biosci, 108:245-251, 1992.

[12] J.C. Cleary. Logical arithmetic. Future Computing Systems, 2(2):125-149, 1987.

[13] E. Davis. Constraint propagation with interval labels. Artificial Intelligence, 32(3):281-331, 1987.

[14] L. Denis-Vidal, G. Joly-Blanchard, and C. Noiret. Some effective approaches to check the identifiability of uncontrolled nonlinear systems. Mathematics and Computers in Simulation, 57:35-44, 2001.

[15] C. Durieu and E. Walter. Estimation ellipsoidales à erreur bornée. In Identification des systèmes, Paris: Hermès, 2001.

[16] C. Jauberthie, N. Verdière, and L. Travé-Massuyès. Setmembership identifiability: definitions and analysis. In Proceedings of the 18th IFAC World Congress, pages 12024-12029, Milan, Italie, 2011

[17] L. Jaulin, M. Kieffer, O. Didrit, and E. Walter. Applied Interval Analysis, with examples in parameter and state estimation, Robust control and robotics. Springer, Londres, 2001.

[18] L. Jaulin and E. Walter. Set inversion via interval analysis for nonlinear bounded-error estimation. Automatica, 29:10531064, 1993.

[19] G. Joly-Blanchard and L. Denis-Vidal. Some remarks about an identifiability result of nonlinear systems. Automatica, 34:11511152, 1998.

[20] M. Kieffer, L. Jaulin, and E. Walter. Guaranteed recursive nonlinear state bounding using interval analysis. International Journal of Adaptative Control and Signal Processing, 6:191-218, 2002.

[21] M. Kieffer and E. Walter. Interval analysis for guaranteed nonlinear parameter estimation. In H.P. Wynn A.C. Atkinson, L. Pronzato, editor, Advances Model-Oriented Data Analysis and Experimental Design, pages 115-125. Physica-Verlag, Heidelberg, 1998.

[22] E.R. Kolchin. Differential algebra and algebraic groups. Academic Press, New York, 1973.

[23] S. Lagrange, N. Delanoue, and L. Jaulin. On sufficient conditions of injectivity, development of a numerical test via interval analysis. Journal Reliable computing, 13:409-421, 2007.

[24] S. Lesecq, A. Barraud, and K. Tran Dinh. Numerical accurate computations for ellipsoidal state bounding. In Proceedings of MED'03, Rhodes, Greece, 2003.

[25] A. Levant. Robust exact differentiation via sliding mode tech- 
nique. Automatica, 34:379-384, 1998.

[26] A. Levant. Higher order sliding modes and arbitrary order exact robust differentiation. In European Control Conference, pages 996-1001, Porto, Portugal, 2001.

[27] A. Levant. Higher-order sliding modes, differentiation and output-feedback control. Int J. Control, 76:924-941, 2003.

[28] L. Ljung and T. Glad. On global identifiability for arbitrary model parametrizations. Automatica, 30:265-276, 1994.

[29] M. Milanese and A. Vicino. Estimation theory for nonlinear models and set membership uncertainty. Automatica, 27(2):403-408, 1991.

[30] R.E. Moore. Interval Analysis. Prentice Hall, New Jersey, 1966.

[31] J. R. Munkres. Topology a first courses. Prentice Hall, New Jersey, 1975

[32] F. Ollivier. Le problème de l'identifiabilité structurelle globale : approche théorique, méthodes effectives et bornes de complexité. PhD, Ecole Polytechnique, France, 1990.

[33] H. Pohjanpalo. System identifiability based on the power series expansion of the solution. Math. Biosciences, 41:21-33, 1978.

[34] T. Raïssi, N. Ramdani, and Y. Candau. Set membership state and parameter estimation for systems described by nonlinear differential equations. Automatica, 40:1771-1777, 2004.

[35] P. Ribot, C. Jauberthie, and L. Travé-Massuyès. State estimation by interval analysis for a nonlinear differential aerospace model. In Proceedings of European Control Conference, pages 48394844, Kos, Greece, 2007.

[36] M.P. Saccomani, S. Audoly, and L. D'Angio. Parameter identifiability of nonlinear sustems: the role of initial conditions. Automatica, 39:619-632, 2004.

[37] R. Seydou, T. Raïssi, A. Zolghadri, D. Efimov, and C. Combastel. Robust fault diagnosis based on constraint satisfaction and interval continuous-time parity equations. In 8th IFAC Symposium on Fault Detection, pages 1293-1298, Mexico City, Mexico, 2012.

[38] N. Verdière, L. Denis-Vidal, G. Joly-Blanchard, and D. Domurado. Identifiability and estimation of pharmacokinetic parameters for the ligands of the macrophage mannose receptor. Int. J. Appl. Math. Comput. Sci., 15:517-526, 2005.

[39] N. Verdière, C. Jauberthie, and L. Travé-Massuyès. Set membership identifiability of nonlinear models. LAAS Report 13001, pages 1-8, January 2013.

[40] E. Walter. Identifiability of state space models. Springer, Berlin, 1994.

[41] E. Walter and Y. Lecourtier. Global approaches to identifiability testing for linear and nonlinear state space models. Math. and Comput.in Simul., 24:472-482, 1982.

[42] D. Waltz. Generating semantic descriptions from drawings of scenes with shadows. The Psychology of Computer Vision, pages 19-91, 1975.

Carine Jauberthie is Associate Professor at University Paul Sabatier of Toulouse (France) since 2005. She is a researcher at the CNRS Laboratoire d'Analyse et d'Architecture des Systèmes (LAAS) (http://www.laas.fr) in the Diagnosis and Supervisory Control (DISCO) research team (http://www.laas.fr/DISCO/). She obtained a Ph.D. degree in 2002 in Applied Mathematics, specialized in system control, from University of Technology of Compiègne, France, at ONERA Center of Lille in collaboration with the Laboratory of Applied Mathematics. From 2002 to 2005, she was one year post-doc at Ecole Centrale of Lille (team SyNer) and she taught Mathematics at Ecole des Mines de Douai.
Her research interests concern fault detection and diagnosis based on interval analysis, the use of constraint satisfaction approaches, and the analysis of the related properties, including identifiability of linear and nonlinear systems.

Nathalie Verdière is Associate Professor at University of Le Havre (France) since 2006. She is a researcher at the Laboratory of Applied Mathematics of Le Havre (http://www.lmah.univ-lehavre.fr) in the "Dynamical Systems" research team (http://www.lmah.univlehavre.fr/systemes_dynamiques). She obtained a Ph.D. degree in 2005 in Applied Mathematics, specialized in system control, from University of Technology of Compiègne, France. From 2005 to 2006, she was a temporary Associate Professor at University of Technology of Compiègne. Her research interests concern bifurcations, identifiability analysis and parameter estimation for models described by ordinary or partial differential equations.

Louise Travé-Massuyès is a Research Director of the Centre National de Recherche Scientifique (CNRS), working at Laboratoire d'Analyse et d'Architecture des Systèmes (LAAS) (http://www.laas.fr), Toulouse, France, where she leads the Diagnosis and Supervisory Control (DISCO) research team (http://www.laas.fr/DISCO/). She received a Ph.D. degree in control in 1984 and an Engineering Degree specialized in control, electronics and computer science in 1982, both from the Institut National des Sciences Appliquées (INSA); then an Habilitation à Diriger des Recherches from Paul Sabatier University in 1998, all in Toulouse, France. Her main research interests are in qualitative and model-based reasoning and applications to dynamic systems supervision and diagnosis. She has been particularly active in bridging the AI and Control Model-Based Diagnosis communities, initially as leader of the BRIDGE Task Group ăof the MONET European Network. She is a member of the IFAC Safeprocess Technical Committee and Senior Member of the IEEE Computer Society. 